\title{
Vernetzung professionellen Wissens angehender Lehrkräfte im Lehramtsstudium
}

\author{
Thilo Kleickmann • Ilonca Hardy
}

Online publiziert: 30. Dezember 2018

(C) Die Herausgeber und Springer Fachmedien Wiesbaden GmbH, ein Teil von Springer Nature 2018

Die Unterscheidung in Fachwissen, fachdidaktisches Wissen und pädagogisch/ psychologisches bzw. fachübergreifendes bildungswissenschaftliches Wissen ist in der Forschung zur professionellen Kompetenz von Lehrkräften weitgehend etabliert (z.B. Baumert und Kunter 2006; König et al. 2016; Krauss et al. 2017). Diese drei Bereiche professionellen Wissens spiegeln sich auch in den zentralen curricularen Inhalten des Lehramtsstudiums in Deutschland wie auch in vielen anderen Ländern wider: Fachliche, fachdidaktische und pädagogisch/psychologische bzw. fachübergreifende bildungswissenschaftliche Anteile stellen typischerweise neben schulpraktischen Anteilen die Kernelemente eines Lehramtsstudiums dar (Bellenberg und Thierack 2003; Terhart 2009). Allerdings sind diese Studienanteile häufig wenig aufeinander abgestimmt und auch innerhalb der Studienanteile wird ein Defizit an Kohärenz und Vernetzung mit schulpraktischen Erfahrungen und unterrichtsbezogenem Wissen konstatiert (Ball 2000; Blömeke 2006; Harr et al. 2014). Vor diesem Hintergrund wird vermutet, dass sich solche inhaltlich wenig verbundenen Lerngelegenheiten auch in fragmentiertem professionellem Wissen der angehenden Lehrkräfte niederschlagen, welches dann im Unterricht nicht angewendet werden könne, also träge bleibe (z.B. Harr et al. 2014). Auch aus der Perspektive von Studierenden stellt die mangelnde Vernetzung zwischen den Studienbestandteilen und innerhalb dieser ein deutliches Desiderat des Lehr-

\footnotetext{
T. Kleickmann $(\bowtie)$

Institut für Pädagogik, Abteilung Schulpädagogik, Christian-Albrechts-Universität zu Kiel,

Olshausenstr. 75, 24118 Kiel, Deutschland

E-Mail: kleickmann@paedagogik.uni-kiel.de

I. Hardy

Institut für Pädagogik der Elementar- und Primarstufe, Arbeitsbereich Grundschulpädagogik mit Schwerpunkt Empirische Bildungsforschung, Goethe-Universität Frankfurt am Main, Theodor-W.-Adorno-Platz 6, 60323 Frankfurt am Main, Deutschland E-Mail: hardy@em.uni-frankfurt.de
} 
amtsstudiums dar (z.B. Blömeke et al. 2006; Mayer et al. 2018). Es verwundert daher nicht, dass die Vernetzung professionellen Wissens ein zentrales Thema in den meisten Projekten der BMBF-geförderten Qualitätsoffensive Lehrerbildung ist: Etwa 35 der 51 teilnehmenden Universitäten erproben Maßnahmen, die auf eine Verbesserung der Vernetzung professionellen Wissens abzielen (z. B. Brouer et al. 2018; Meier et al. 2018).

\section{Vernetzung professionellen Wissens durch Lerngelegenheiten}

Die Vernetzung professionellen Wissens angehender Lehrkräfte wird durch sehr unterschiedliche Formen der Vernetzung von Lerngelegenheiten im Rahmen der Lehrerinnen- und Lehrerbildung angestrebt (z. B. Mayer et al. 2018). Mit der horizontalen Vernetzung sind $\mathrm{u}$.a. die Vernetzung der fachlichen, fachdidaktischen und fachübergreifenden bildungswissenschaftlichen Studienbestandteile, die Vernetzung des Wissens innerhalb dieser Bereiche und die Vernetzung mit schulpraktischen Elementen gemeint. Die vertikale Vernetzung bezieht sich vor allem auf die Vernetzung der Institutionen und Lerngelegenheiten zwischen den drei Phasen der Lehrerinnenund Lehrerbildung. Vernetzung kann außerdem realisiert werden als strukturelle (u. a. über Modulbeschreibungen verstetigt) oder kollegiale Vernetzung durch Kooperation der Lehrenden. Bei Mayer et al. (2018) findet sich eine hilfreiche Darstellung verschiedener Modelle zur Vernetzung von fachlichen, fachdidaktischen und pädagogisch/psychologischen Lerngelegenheiten im Rahmen der Lehrerinnen- und Lehrerbildung.

Aus kognitionspsychologischer Sicht wird der Begriff des vernetzten Wissens verwendet, um eine elaborierte und gut strukturierte Wissensbasis zu beschreiben, welche in unterschiedlichen Situationen zur Anwendung kommt (Renkl 1996). In Bezug auf Lehrkräfte hat Bromme (1992) darauf hingewiesen, dass sich deren Expertise durch ein vernetztes, gut organisiertes professionelles Wissen auszeichnet. Zwischen vernetzten Lerngelegenheiten und vernetztem Wissen wird ein enger Zusammenhang angenommen. So zielen Maßnahmen zur Vernetzung der Lerngelegenheiten in der Lehramtsausbildung darauf ab, die drei Wissensbereiche sinnvoll aufeinander zu beziehen und darüber die Anwendbarkeit und die von den Studierenden wahrgenommene Berufsrelevanz des Wissens zu erhöhen (vgl. Ball 2000; Mayer et al. 2018).

\section{Forschung zur Vernetzung professionellen Wissens}

Der Stand der Forschung zum professionellen Wissen von angehenden Lehrkräften ist in den vergangenen 15 Jahren deutlich erweitert worden, insbesondere im Hinblick auf die Erfassung und Struktur professionellen Wissens, die Ausprägung und Entwicklung des Wissens wie auch im Hinblick auf die Bedingungen, die zu einem Aufbau dieses Wissens beitragen (z. B. König et al. 2016; Krauss et al. 2017). Allerdings ist in den bislang vorliegenden Studien die Annahme, vernetztes fachliches, fachdidaktisches und pädagogisch/psychologisches Wissen begünstige die Anwend- 
barkeit des Wissens in realen unterrichtlichen oder unterrichtsnahen Anforderungssituationen, noch kaum untersucht worden (z. B. Meier et al. 2018). Insgesamt ist der Forschungsstand zu Fragen der Vernetzung des professionellen Wissens noch als wenig zufriedenstellend zu bezeichnen (z. B. Evens et al. 2018).

Erste Studien haben jedoch bereits Erkenntnisse darüber geliefert, inwiefern sich Lerngelegenheiten, in denen bestimmte Bereiche des professionellen Wissens vernetzt gelehrt werden, positiv auf die Entwicklung der einzelnen Bereiche des professionellen Wissens angehender Lehrkräfte und auf die Vernetzung des Wissens auswirken (Evens et al. 2018; Harr et al. 2014; Wäschle et al. 2015). Evens et al. (2018) fanden hier beispielsweise, dass das fachdidaktische Wissen angehender Lehrkräfte durch vernetzte Lerngelegenheiten für fachliches, fachdidaktisches und pädagogisches Wissen nicht besser gefördert wurde als durch die separat gelehrten Wissensbereiche. Harr et al. (2014) konnten hingegen Vorteile einer integrierten (vs. separaten) Darbietung fachdidaktischen und pädagogisch/psychologischen Wissens für die Entwicklung fachdidaktischen Wissens nachweisen.

Weitere Studien untersuchten, wie sich das verfügbare Wissen in einem Bereich des professionellen Wissens auf andere Bereiche des professionellen Wissens auswirkt. Solche Effekte zwischen den Bereichen des professionellen Wissens sind plausibel, da Fachwissen und fachdidaktisches Wissen die fachlichen Inhalte als Überlappungsbereich aufweisen und fachdidaktisches und pädagogisch/psychologisches Wissen das Wissen über Lehren und Lernen. Diese spezifischen Überlappungen bzw. gemeinsamen Elemente spiegeln sich zunächst in den korrelativen Beziehungen zwischen den drei Wissensbereichen wider. Fachwissen und fachdidaktisches Wissen bzw. fachdidaktisches Wissen und pädagogisch/psychologisches Wissen korrelieren i.d.R. stärker als Fachwissen und pädagogisch/psychologisches Wissen (z. B. König et al. 2016; Krauss et al. 2008). Einige Befunde deuten außerdem darauf hin, dass die Korrelationen zwischen den Wissensbereichen mit zunehmender Expertise steigen, was hier als zunehmende Vernetzung des Wissens interpretiert wird (Krauss et al. 2008). Die Befundlage ist diesbezüglich aber nicht ganz einheitlich.

Über korrelative Studien hinaus wurde in ersten experimentellen Studien untersucht, wie sich ein durch Lerneinheiten verursachter Zugewinn an professionellem Wissen im einen Wissensbereich auf das professionelle Wissen in anderen Wissensbereichen auswirkt, wenn die Wissensbereiche eng aufeinander abgestimmt sind (z. B. Evens et al. 2018; Tröbst et al. 2018). Tröbst et al. (2018) fanden hier in einer Studie mit angehenden Grundschullehrkräften, dass die Förderung des Fachwissens zu einem gewissen Grad hinreichend für die Entwicklung fachdidaktischen Wissens ist. Im Verhältnis zu den Lerneffekten im Rahmen einer expliziten Vermittlung fachdidaktischen Wissens waren die Effekte allerdings eher klein. Durch rein pädagogische Lerneinheiten verursachte Zugewinne im pädagogischen Wissen führten zu keiner Verbesserung des fachdidaktischen Wissens.

Andere Studien untersuchten, ob sich bei Lehramtsstudierenden ein weiteres Fach aus einer Fächergruppe positiv auf die Entwicklung fachlichen und fachdidaktischen Wissens auswirkt. In einer Studie mit angehenden Biologie-Lehrkräften (Großschedl et al. 2015) zeigte sich, dass Studierende, die als Zweitfach eine weitere Naturwissenschaft (Physik oder Chemie) studierten, bessere Werte im biologiedidaktischen Wissen, nicht jedoch im Fachwissen aufwiesen. 
Diskussionen zur Vernetzung professionellen Wissens betreffen nicht nur den Aufbau entsprechenden Wissens unter Annahme der Bedeutsamkeit von fachdidaktischem, fachlichem und pädagogisch/psychologischem Wissen. Sie betreffen auch die Frage der Ausdifferenzierung von fachlichen Wissensstrukturen. So liegen im Zusammenhang mit der Vernetzung von universitärem und schulnahem professionellen Wissen innerhalb des Fachwissens eine Reihe von Vorschlägen zur Erweiterungen bzw. Ausdifferenzierung dieses Wissensbereichs vor. Ball, Thames und Phelps (2008) schlagen hierzu die Bereiche ,horizon content knowledge“, „,common content knowledge“ und „specialized content knowledge“ (S. 403) vor, die sich u.a. im Hinblick auf ihre Nähe zu schulischen Inhalten unterscheiden. Dreher, Lindmeier, Heinze und Niemand (2018) legen ein Modell vor, in dem das fachbezogene professionelle Wissen um eine Komponente des Fachwissens im schulischen Kontext erweitert wird. Dieses Konstrukt beschreibt berufsspezifisches Fachwissen von Mathematiklehrkräften über Zusammenhänge zwischen universitärer und schulischer Mathematik.

\section{Erweiterung des Forschungsstandes durch die Beiträge in diesem Themenheft}

Die vier Beiträge des vorliegenden Themenhefts stellen empirische Studien vor, die den Stand der Forschung zur Vernetzung professionellen Wissens um wichtige Befunde erweitern.

Ausgangspunkt für den Beitrag von Graichen, Wegner und Nückles ist die Tatsache, dass Fachwissen, fachdidaktisches Wissen und pädagogisch/psychologisches Wissen meist in kaum verbundenen Lerngelegenheiten im Lehramtsstudium vermittelt werden. Graichen et al. gehen daher davon aus, dass auch das professionelle Wissen von Lehramtsstudierenden fragmentiert ist und in Anwendungssituationen träge bleibt. In einer experimentellen Studie wird untersucht, inwiefern durch bestimmte Unterstützungsmaßnahmen (Modellierung anhand von Lösungsbeispielen und Leitfragen) beim Schreiben von Lerntagebüchern im Rahmen einer onlinebasierten Lernumgebung der Erwerb kohärenzbildendender Lernstrategien und die Anwendung professionellen Wissens bei Lehramtsstudierenden $(N=74)$ im Fach Geografie unterstützt werden kann. Es zeigt sich u.a., dass die Unterstützungsmaßnahmen zwar nicht zu einem höheren Niveau des professionellen Wissens geführt, wohl aber dessen Nutzung in unterrichtsnahen Anwendungsaufgaben verbessert haben. Die Ergebnisse implizieren, dass Lerntagebücher mit geeigneter instruktionaler Unterstützung die Kohärenzbildung im professionellen Wissen und die Nutzung des Wissens bei unterrichtsbezogenen Anwendungsaufgaben verbessern können.

Den Ausgangspunkt für die Studie von Lorentzen, Friedrichs, Ropohl und Steffensky bildet die Schwierigkeit von Lehramtsstudierenden, die Relevanz universitärer fachlicher Inhalte für ihre spätere Berufstätigkeit als Lehrkraft zu erkennen. Lorentzen et al. haben daher eine Intervention entwickelt, die die Studierenden durch die Erstellung von Wissensnetzen darin unterstützen soll, universitäres und schulisches Fachwissen zu verknüpfen und darüber die Berufsrelevanz des fachlichen Wissens besser erkennen zu können. In einer experimentellen Studie mit Che- 
mielehramtsstudierenden $(N=55)$ haben Lorentzen et al. die Intervention in eine reguläre Fachveranstaltung zur physikalischen Chemie integriert und diese Veranstaltung mit einer entsprechenden Veranstaltung ohne Maßnahmen zur Vernetzung kontrastiert. Die Ergebnisse zeigen, dass die Vernetzungs-Intervention die wahrgenommene Berufsrelevanz der vermittelten Inhalte verbessert hat. Gleichzeitig gehen die Maßnahmen zur Vernetzung nicht auf Kosten des fachlichen Lernzuwachses. Gleichwohl weisen qualitative Analysen auf bestimmte Defizite in den Begründungen der Berufsrelevanz hin.

Die Studie von Paetsch, Darsow, Wagner, Hammer und Ehmke adressiert den im Hinblick auf professionelles Wissen und professionelle Kompetenz angehender Lehrkräfte erst in Ansätzen untersuchten Bereich Deutsch als Zweitsprache (DaZ). Im Mittelpunkt der Studie steht die Frage nach den Bedingungen des Kompetenzerwerbs im Rahmen eines DaZ-Moduls. Insbesondere wird aufgrund von Überlappungen in den Lerngelegenheiten der Frage nachgegangen, ob Lehramtsstudierende mit einer Fremdsprache als Unterrichtsfach oder dem Unterrichtsfach Deutsch einen größeren Zuwachs an DaZ-Kompetenz aufweisen als Studierende ohne diese Fächer. In der Studie mit Lehramtsstudierenden $(N=180)$ verschiedener Fächer und Schulformen zeigt sich u. a., dass Studierende mit einer Fremdsprache, nicht jedoch Studierende mit dem Fach Deutsch, einen größeren Kompetenzzuwachs im Rahmen des DaZ-Moduls haben. Lerngelegenheiten in der Fremdsprache scheinen also die Nutzung der DaZ-Lerngelegenheiten verbessert zu haben.

Der Beitrag von Tröbst, Kleickmann, Depaepe, Heinze und Kunter greift die Tatsache auf, dass es zwischen dem fachdidaktischen Wissen und dem Fachwissen sowie zwischen dem fachdidaktischen und dem pädagogischen Wissen spezifische Überlappungen gibt. Tröbst et al. gehen hier der Frage nach, ob eine Förderung des fachdidaktischen Wissens aufgrund dieser Überlappungen zudem gewissermaßen ,beiläufig“ zu einer Förderung des Fachwissens und des pädagogischen Wissens beiträgt. Untersucht wird diese Frage in einer experimentellen Studie mit 59 Studierenden (Grundschullehramt, Fach Mathematik). Es zeigen sich kleine Effekte der Förderung des fachdidaktischen Wissens auf das pädagogisch/psychologische Wissen und auf das Fachwissen. Die Ergebnisse deuten darauf hin, dass die Förderung fachdidaktischen Wissens auch positive Auswirkungen auf eng mit diesem Wissen abgestimmte Bereiche des generischen pädagogisch/psychologischen Wissens und des Fachwissens haben kann, die im Rahmen des Lehramtsstudiums gezielt genutzt werden können.

Insgesamt wird mit den Beiträgen dieses Themenhefts die Befundlage zu instruktionalen Kontexten erweitert, welche eine Vernetzung von Wissensbereichen des professionellen Wissens von Lehramtsstudierenden bewirken können. Es wird außerdem aufgezeigt, welche Wissenszuwächse sich in unterschiedlichen Fächern durch Überlappungen zwischen Inhalten ergeben können. Durch einen hohen Grad an curricularer Anbindung sind die vorliegenden Befunde für die Lehramtsausbildung äußerst relevant und es stellt sich als vielversprechend dar, in weiteren Studien die Auswirkungen des Grads an Überlappung von Studieninhalten und Möglichkeiten der Kohärenzbildung vertiefend zu behandeln. 


\section{Literatur}

Ball, D. L. (2000). Bridging practices: intertwining content and pedagogy in teaching and learning to teach. Journal of Teacher Education, 51, 241-247. https://doi.org/10.1177/0022487100051003013.

Ball, D.L., Thames, M., \& Phelps, G. (2008). Content knowledge for teaching. What makes it special? Journal of Teacher Education, 59, 389-407. https://doi.org/10.1177/0022487108324554.

Baumert, J., \& Kunter, M. (2006). Stichwort: Professionelle Kompetenz von Lehrkräften. Zeitschrift für Erziehungswissenschaft, 9, 469-520. https://doi.org/10.1007/s11618-006-0165-2.

Bellenberg, G., \& Thierack, A. (2003). Ausbildung von Lehrerinnen und Lehrern in Deutschland: Bestandsaufnahme und Reformbestrebungen. Wiesbaden: Springer.

Blömeke, S. (2006). Struktur der Lehrerausbildung im internationalen Vergleich. Ergebnisse einer Untersuchung in acht Ländern. Zeitschrift für Pädagogik, 52, 393-416.

Blömeke, S., Müller, C., \& Felbrich, A. (2006). Forschung - Theorie - Praxis. Einstellungen von Studierenden und Referendaren zur Lehrerausbildung. Die deutsche Schule, 98(2), 178-189.

Bromme, R. (1992). Der Lehrer als Experte. Zur Psychologie des professionellen Wissens. Bern: Huber.

Brouer, B., Burda-Zoyke, A., Kilian, J., \& Petersen, I. (Hrsg.). (2018). Vernetzung in der Lehrerinnenund Lehrerbildung. Ansätze, Methoden und erste Befunde aus dem LeaP-Projekt an der ChristianAlbrechts-Universität zu Kiel. Münster: Waxmann.

Dreher, A., Lindmeier, A., Heinze, A., \& Niemand, C. (2018). What kind of content knowledge do secondary mathematics teachers need? Journal für Mathematik-Didaktik, 39, 319-341. https://doi.org/10. 1007/s13138-018-0127-2.

Evens, M., Elen, J., Larmuseau, C., \& Depaepe, F. (2018). Promoting the development of teacher professional knowledge: integrating content and pedagogy in teacher education. Teaching and Teacher Education, 75, 244-258. https://doi.org/10.1016/j.tate.2018.07.001.

Großschedl, J., Harms, U., Kleickmann, T., \& Glowinski, I. (2015). Preservice biology teachers' professional knowledge: structure and learning opportunities. Journal of Science Teacher Education, 26, 291-318. https://doi.org/10.1007/s10972-015-9423-6.

Harr, N., Eichler, A., \& Renkl, A. (2014). Integrating pedagogical content knowledge and pedagogical/ psychological knowledge in mathematics. Frontiers in Psychology, 5, 1-10. https://doi.org/10.3389/ fpsyg.2014.00924.

König, J., Lammerding, S., Nold, G., Rohde, A., Strauß, S., \& Tachtsoglou, S. (2016). Teachers' professional knowledge for teaching English as a foreign language: assessing the outcomes of teacher education. Journal of Teacher Education, 67, 320-337. https://doi.org/10.1177/0022487116644956.

Krauss, S., Lindl, A., Schilcher, A., \& Tepner, O. (2017). Das Forschungsprojekt FALKO - ein einleitender Überblick. In S. Krauss, A. Lindl, A. Schilcher, M. Fricke, A. Göhring, B. Hofmann \& R. Mulder, et al. (Hrsg.), FALKO - Fachspezifische Lehrerkompetenzen. Konzeption von Professionswissenstests in den Fächern Deutsch, Englisch, Latein, Physik, Musik, Evangelische Religion und Pädagogik (S. 9-66). Münster: Waxmann.

Krauss, S., Brunner, M., Kunter, M., Baumert, J., Blum, W., Neubrand, J., \& Jordan, A. (2008). Pedagogical content knowledge and content knowledge of secondary mathematics teachers. Journal of Educational Psychology, 100, 716-725. https://doi.org/10.1037/0022-0663.100.3.716.

Mayer, J., Ziepprecht, K., \& Meier, M. (2018). Vernetzung fachlicher, fachdidaktischer und bildungswissenschaftlicher Studienelemente in der Lehrerbildung. In M. Meier, K. Ziepprecht \& J. Mayer (Hrsg.), Lehrerausbildung in vernetzten Lernumgebungen (S. 9-20). Münster: Waxmann.

Meier, M., Ziepprecht, K., \& Mayer, J. (Hrsg.). (2018). Lehrerausbildung in vernetzten Lernumgebungen. Münster: Waxmann.

Renkl, A. (1996). Träges Wissen: Wenn Erlerntes nicht genutzt wird. Psychologische Rundschau, 47, 78-92.

Terhart, E. (2009). Erste Phase: Lehrerbildung an der Universität. In O. Zlatkin-Troitschanskaia, K. Beck, D. Sembill, R. Nickolaus \& R. Mulder (Hrsg.), Lehrprofessionalität: Bedingungen, Genese, Wirkungen und ihre Messung (S. 425-435). Weinheim: Beltz.

Tröbst, S., Kleickmann, T., Heinze, A., Anschütz, A., Rink, R., \& Kunter, M. (2018). Teacher knowledge experiment: Testing mechanisms underlying the formation of preservice elementary school teachers' pedagogical content knowledge concerning fractions and fractional arithmetic. Journal of Educational Psychology, 110, 1049-1065. https://doi.org/10.1037/edu0000260.

Wäschle, K., Gebhard, A., Oberbusch, E.-M., \& Nückles, M. (2015). Journal writing in science: effects on comprehension, interest, and critical reflection. Journal of Writing Research, 7(1), 41-64. https://doi. org/10.17239/jowr-2015.07.01.03. 